Opinion

\section{Entropy via Art}

\section{Abraham Tamir* \\ Emeritus Professor, Israel}

From the energy involved in a physical or chemical process, part of it cannot be utilised as work. Entropy $S$ is the thermodynamic quantity that is the measure of this energy where the concept of entropy was first introduced by Rudolf Clausius (1822-1888) in 1865. Entropy is also a basic and general conception that deals with "order" (low entropy) and "disorder" (high entropy) of all systems in the universe from human body and up to the star systems. With the development of science entropy deviates from treatment of subjects related to heat and work and currently it is used also to analyse knowledge as well as in economics.

In the following we demonstrate the concept of entropy by artworks. The paintings of the Dutch artist Piet Mondrian (1872-1944) on the left column are compositions in three colours: red, yellow and blue. At the bottom picture parallel lines are described that make an impression of an ordered system, namely of a relatively low entropy. By going up the pictures, the parallel lines vanish and a mixture of squares and rectangles are added that spoil the order. In thermodynamic terms it can be said that the entropy in the work of art is increasing. The photographs of the American Walter Wick describe the three states of water. In ice the molecules are arranged in order and the entropy is low. In the liquid water the attraction forces between the molecules become weaker and the molecules can move. In the transition to this state the disorder, namely the entropy, increases. During boiling the molecules gain free motion, and the disorder increases and the entropy is higher. The three additional paintings of the American artist Walter Wick (b.1953) demonstrate a drop of color that is introduced into water. The molecular diffusion processes disperse the colour molecules in all the volume of the vessel until a uniform dispersion in which the entropy is the highest.

The work of art "The Three Ages of Man" (c.1510) on the right-hand-side by the German artist Hans Baldung (c.14801545) demonstrates the concept of entropy also in man's life. By the passing of time, demonstrated by the sand clock in the hand of death in a form of a skeleton, the sleeping baby girl will grow up to a young woman that the end will become old and die. The low entropy in the baby's body that her body systems are in order and normal, will increase as she becomes older and will spoil the interaction between her body organs.

\author{
More Information \\ *Address for Correspondence: Abraham Tamir \\ Emeritus Professor, Israel, \\ Email: atamir4@012.net.il \\ Submitted: 03 June 2020 \\ Approved: 12 August 2020 \\ Published: 13 August 2020 \\ How to cite this article: Tamir A. \\ Entropy via Art. Int J Phys Res Appl. 2020; 3: \\ 123-123. \\ DOI: 10.29328/journal.ijpra.1001029 \\ Copyright: (c) 2020 Tamir A. This is an open \\ access article distributed under the Creative \\ Commons Attribution License, which permits \\ unrestricted use, distribution, and reproduction \\ in any medium, provided the original work is \\ properly cited. \\ D) Check for updates \\ OPen access
}

When the body will reach maximum entropy it will stop to perform and the woman will die. Combination of faces demonstrates the final example of entropy. The first kind is facial combinations of Leonardo Da Vinci (down image) and his artwork Mona Lisa (top image). The original faces may be considered of the highest order - minimal entropy - where that at the center, of the highest disorder, may be considered of maximal entropy. The other two, of middle order, may be considered of middle entropy. In conclusion the authors believe that the presentation of entropy by different artworks and face combinations makes this thermodynamic function more understandable and perceptible.

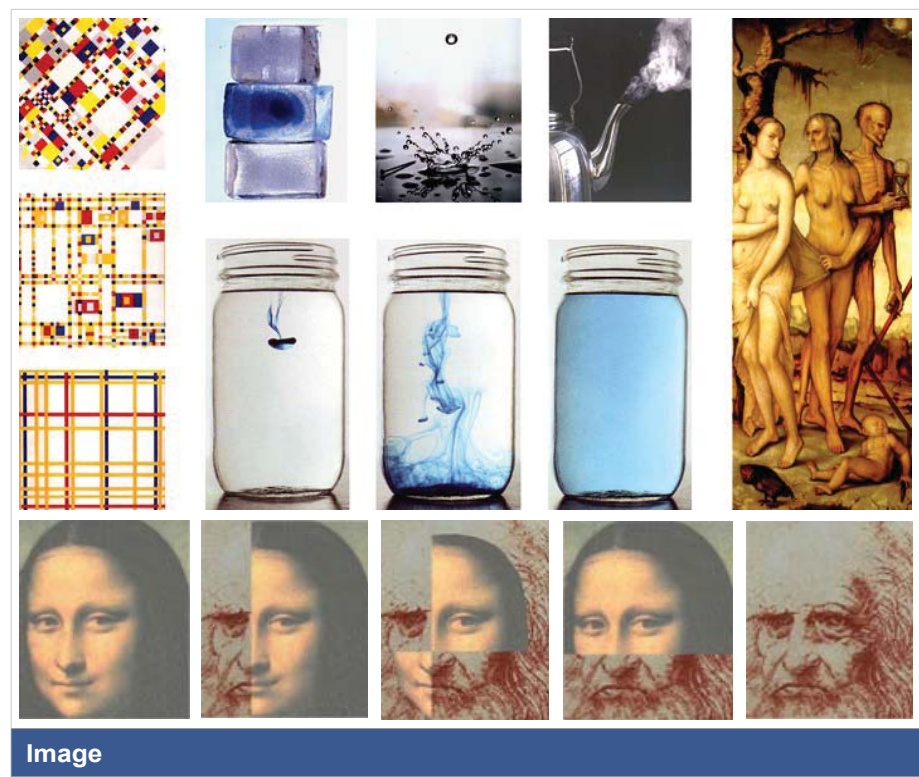

